\title{
THE NONLINEAR CIRCULAR MEMBRANE UNDER A VERTICAL FORCE*
}

\author{
BY \\ R. W. DICKEY \\ University of Wisconsin, Madison
}

\begin{abstract}
The exact theory for the deformation of a plane circular membrane under a vertical force is derived. It is shown that the system of equations can be reduced to a single, nonlinear, ordinary differential equation. In addition it is shown that the Foppl approximation is the first term in an asymptotic expansion of the exact theory.
\end{abstract}

1. Introduction. In 1907 Foppl [1] suggested an approximate theory for the study of certain plane membrane problems. This theory has proved to be of particular interest in studying the radially symmetric deformation of a plane circular membrane under a vertical force. In this case the Foppl equations can be reduced to a boundary value problem for single, nonlinear ordinary differential equation with the radial stress as the quantity to be determined [2]. This equation has been studied in detail in [3-5].

One problem with the Foppl theory is the ad hoc nature of its derivation. Thus, even though the Foppl approximations are physically plausible, there is not even a formal mathematical justification. Indeed it is not clear in what sense, if any, the Foppl theory approximates the exact theory nor is it obvious how to proceed in a systematic way to obtain better approximations. In this paper we will show that the Foppl theory is the first term in an asymptotic expansion of the exact theory and in addition, we will indicate the procedure for obtaining higher order approximations.

The equations governing the radially symmetric deformation of a plane circular elastic surface under a vertical pressure $P$ (for simplicity we assume $P$ is constant) is a combination of three sets of relations-(1) the strain-displacement equations, (2) the constitutive equations, (3) the equilibrium equations:

$$
\begin{aligned}
\varepsilon_{r} & =\left[\left(1+u^{\prime}\right)^{2}+\left(w^{\prime}\right)^{2}\right]^{1 / 2}-1, \\
\varepsilon_{\theta} & =u / r, \\
\sigma_{r} & =\sigma_{r}\left(\mathcal{E}_{r}, \varepsilon_{\theta}\right), \\
\sigma_{\theta} & =\sigma_{\theta}\left(\mathcal{E}_{r}, \mathcal{E}_{\theta}\right),
\end{aligned}
$$

${ }^{*}$ Received October 6, 1982. This research was supported by the National Research Foundation, Grant No. 75-06366. 


$$
\begin{gathered}
\frac{d}{d r}\left\{\frac{\sigma_{r}(r+u)\left(1+u^{\prime}\right)}{\left[\left(1+u^{\prime}\right)^{2}+\left(w^{\prime}\right)^{2}\right]^{1 / 2}}\right\}-\sigma_{\theta}\left[\left(1+u^{\prime}\right)^{2}+\left(w^{\prime}\right)^{2}\right]^{1 / 2}=0, \\
\frac{d}{d r}\left\{\frac{\sigma_{r}(r+u) w^{\prime}}{\left[\left(1+u^{\prime}\right)^{2}+\left(w^{\prime}\right)^{2}\right]^{1 / 2}}\right\}+\frac{P r}{h}=0
\end{gathered}
$$

$\left(^{\prime}=d / d r\right) . u$ and $w$ are the radial and vertical displacements, $\varepsilon_{r}$ and $\varepsilon_{\theta}$ are the radial and circumferential strains, $\sigma_{r}$ and $\sigma_{\theta}$ are the radial and circumferential stresses, and $h$ is the thickness of the membrane. The Foppl theory assumes Hooke's law. In this case the equations (1.2) become

$$
\begin{aligned}
& \sigma_{r}=\frac{E}{1-\nu^{2}}\left(\varepsilon_{r}+\nu \varepsilon_{\theta}\right), \\
& \sigma_{\theta}=\frac{E}{1-\nu^{2}}\left(\varepsilon_{\theta}+\nu \varepsilon_{r}\right)
\end{aligned}
$$

where $E$ is Young's modulus and $\nu$ is the Poisson ratio. For the boundary conditions on (1.1), (1.2) and (1.3) we prescribe either radial displacement (the displacement problem)

$$
u(a)=\mu
$$

or the radial stress (the stress problem)

$$
\sigma_{r}(a)=\boldsymbol{\sigma}
$$

( $a$ is the radius of the undeformed membrane). We also assume that $w(a)=0$ and all quantities remain finite at $r=0$.

In Sec. 2 it will be shown that the system of equations (1.1), (1.2), and (1.3) can be reduced to a single ordinary differential equation. In Sec. 3 we will obtain the Foppl theory as the first term in an asymptotic expansion of the exact theory and we will indicate how to obtain higher order approximations. In the appendix (Sec. 4) the equations (1.1), (1.2), and (1.3) are derived.

2. Reduction of the equations. The pair of equilibrium equations (1.3) can be rewritten

$$
\begin{array}{r}
\frac{d}{d r}\left\{\frac{r \Sigma_{r}\left(1+u^{\prime}\right)}{1+\varepsilon_{r}}\right\}-\Sigma_{\theta}=0 \\
\frac{d}{d r}\left\{\frac{r \Sigma_{r} w^{\prime}}{1+\varepsilon_{r}}\right\}+\frac{P r}{h}=0
\end{array}
$$

where we have introduced the change of variable

$$
\Sigma_{r}=\left(1+\varepsilon_{\theta}\right) \sigma_{r}, \quad \Sigma_{\theta}=\left(1+\varepsilon_{r}\right) \sigma_{\theta} .
$$

In the case that the constitutive equations are derivable from a strain energy density function, i.e. if there is a function $W\left(\varepsilon_{r}, \varepsilon_{\theta}\right)$ such that

$$
\sigma_{r}=\frac{\partial W}{\partial \varepsilon_{r}}, \quad \sigma_{\theta}=\frac{\partial W}{\partial \varepsilon_{\theta}}
$$


then

$$
\Sigma_{r}=\left(1+\varepsilon_{\theta}\right) \frac{\partial W}{\partial \varepsilon_{r}}, \quad \Sigma_{\theta}=\left(1+\varepsilon_{r}\right) \frac{\partial W}{\partial \varepsilon_{\theta}} .
$$

It will be assumed that this system can be solved for $\mathcal{E}_{r}$ and $\mathcal{E}_{\theta}$ as functions of $\Sigma_{r}$ and $\Sigma_{\theta}$. A sufficient condition would be that the Jacobian

$$
\frac{\partial\left(\left(1+\varepsilon_{\theta}\right) \partial W / \partial \varepsilon_{r},\left(1+\varepsilon_{r}\right) \partial W / \partial \varepsilon_{\theta}\right)}{\partial\left(\varepsilon_{r}, \varepsilon_{\theta}\right)} \neq 0
$$

It is easily verified that if

$$
\frac{\partial^{2} W}{\partial \mathcal{E}_{r}^{2}} \frac{\partial^{2} W}{\partial \mathcal{E}_{\theta}^{2}}-\left(\frac{\partial^{2} W}{\partial \mathcal{E}_{r} \partial \mathscr{E}_{\theta}}\right) \neq 0
$$

when $\varepsilon_{r}=\varepsilon_{\theta}=0$ then (2.5) will be satisfied when the strains are sufficiently small. In any case we assume

$$
\mathcal{E}_{r}=e_{r}\left(\Sigma_{r}, \Sigma_{\theta}\right), \quad \mathcal{E}_{\theta}=e_{\theta}\left(\Sigma_{r}, \Sigma_{\theta}\right)
$$

The equations (2.1) may be integrated to find

$$
\begin{aligned}
\frac{r \Sigma_{r}\left(1+u^{\prime}\right)}{1+\varepsilon_{r}} & =\int_{0}^{r} \Sigma_{\theta} d \tau, \\
\frac{r \Sigma_{r} w^{\prime}}{1+\varepsilon_{r}} & =\frac{P r^{2}}{2 h} .
\end{aligned}
$$

The equations (2.8) may be combined to show that

$$
\begin{aligned}
r \Sigma_{r} & =\left[\frac{P^{2} r^{4}}{4 h^{2}}+\left(\int_{0}^{r} \Sigma_{\theta} d \tau\right)^{2}\right]^{1 / 2}, \\
w^{\prime} & =\frac{-P^{2}\left(1+u^{\prime}\right)}{2 h \int_{0}^{r} \Sigma_{\theta} d \tau} .
\end{aligned}
$$

The choice of the positive root in (2.9) insures that the membrane is in tension.

If equation (2.10) is combined with (1.1a) we find that

$$
\mathcal{E}_{r}+1=e_{r}\left(\Sigma_{r}, \Sigma_{\theta}\right)+1=\frac{\left(1+u^{\prime}\right) r \Sigma_{r}}{\int_{0}^{r} \Sigma_{\theta} d \tau}
$$

where we assume that $u^{\prime}>-1$. Equivalently

$$
1+u^{\prime}=\frac{\int_{0}^{r} \Sigma_{\theta} d \tau}{r \Sigma_{r}}+\frac{e_{r}\left(\Sigma_{r}, \Sigma_{\theta}\right) \int_{0}^{r} \Sigma_{\theta} d \tau}{r \Sigma_{r}}
$$

It is a consequence of $(1.1 \mathrm{~b})$ that

$$
u^{\prime}=\frac{d}{d r} r e_{\theta}\left(\Sigma_{r}, \Sigma_{\theta}\right)
$$


so that

$$
1+\frac{d}{d r} r e_{\theta}\left(\Sigma_{r}, \Sigma_{\theta}\right)=\frac{S}{\Sigma_{r}}+\frac{e_{r}\left(\Sigma_{r}, \Sigma_{\theta}\right) S}{\Sigma_{r}}
$$

where we have introduced the notation

$$
r S=\int_{0}^{r} \Sigma_{\theta} d \tau=\frac{r \Sigma_{r}\left(1+u^{\prime}\right)}{1+\varepsilon_{r}} .
$$

In this notation we have

$$
\Sigma_{r}=\left[\frac{P^{2} r^{2}}{4 h^{2}}+S^{2}\right]^{1 / 2}, \quad \Sigma_{\theta}=\left(r S^{\prime}\right) .
$$

In view of (2.16) it is clear that $(2.14)$ is a second order ordinary differential equation for the determination of $S$.

The boundary condition on $S$ are either

$$
\left.e_{\theta}\left(\left[\frac{P^{2} r^{2}}{4 h^{2}}+S^{2}\right]^{1 / 2},\left(r S^{\prime}\right)^{\prime}\right)\right|_{r=a}=\frac{u(a)}{a}=\frac{\mu}{a}
$$

(cf. (1.5)) or

$$
\left.\frac{\left[P^{2} r^{2} / 4 h^{2}+S^{2}\right]^{1 / 2}}{1+e_{\theta}\left(\left[P^{2} r^{2} / 4 h^{2}+S^{2}\right]^{1 / 2},(r S)^{\prime}\right)}\right|_{r=a}=\sigma
$$

(cf. (1.6)). In either case we require that $S(0)$ is finite.

3. Approximate theories. As was indicated in Sec. 1 Foppl theory assumes the constitutive laws are given by (1.4). These constitutive equations are derivable from the classical strain energy density function

$$
W=\frac{E}{2\left(1-\nu^{2}\right)}\left(\varepsilon_{r}^{2}+\varepsilon_{\theta}^{2}+2 \nu \varepsilon_{r} \varepsilon_{\theta}\right) .
$$

Thus the Lagrange stresses (cf. (2.4)) are

$$
\begin{aligned}
& \Sigma_{r}=\frac{E}{1-\nu^{2}}\left(1+\varepsilon_{\theta}\right)\left(\varepsilon_{r}+\nu \varepsilon_{\theta}\right), \\
& \Sigma_{\theta}=\frac{E}{1-\nu^{2}}\left(1+\varepsilon_{r}\right)\left(\varepsilon_{\theta}+\nu \varepsilon_{r}\right) .
\end{aligned}
$$

Equation (3.2) can be solved for $\varepsilon_{r}$ and $\varepsilon_{\theta}$ to find

$$
\begin{aligned}
\mathcal{E}_{r}= & e_{r}\left(\Sigma_{r}, \Sigma_{\theta}\right) \\
= & \frac{1}{E}\left(\Sigma_{r}-\nu \Sigma_{\theta}\right)+\frac{1}{E^{2}}\left(\nu \Sigma_{r}^{2}-(1-\nu) \Sigma_{r} \Sigma_{\theta}-\nu^{2} \Sigma_{\theta}^{2}\right) \\
& +\vartheta\left(\left[\Sigma_{r}^{2}+\Sigma_{\theta}^{2}\right]^{3 / 2}\right)
\end{aligned}
$$




$$
\begin{aligned}
\mathcal{E}_{\theta}= & e_{\theta}\left(\Sigma_{r}, \Sigma_{\theta}\right) \\
= & \frac{1}{E}\left(\Sigma_{\theta}-\nu \Sigma_{r}\right)+\frac{1}{E^{2}}\left(\nu \Sigma_{\theta}^{2}-(1-\nu) \Sigma_{r} \Sigma_{\theta}-\nu^{2} \Sigma_{r}^{2}\right) \\
& +\vartheta\left(\left[\Sigma_{r}^{2}+\Sigma_{\theta}^{2}\right]^{3 / 2}\right) .
\end{aligned}
$$

It is convenient to introduce the following changes of variable

$$
\begin{gathered}
\rho=r / a, \quad k^{3}=P^{2} a^{2} / 4 h^{2}, \\
S=k T .
\end{gathered}
$$

The parameter $k$ defined in (3.4) is related to a parameter which occurs naturally in the Foppl theory. In any case equation (2.14) becomes

$$
\begin{aligned}
1+\frac{d}{d \rho} \rho e_{\theta}\left(k\left[k \rho^{2}+T^{2}\right]^{1 / 2}, k \frac{d}{d \rho}(\rho T)\right) & \\
& =\frac{T}{\left[k \rho^{2}+T^{2}\right]^{1 / 2}}+\frac{e_{r}\left(k\left[k \rho^{2}+T^{2}\right]^{1 / 2}, d(\rho T) / d \rho\right) T}{\left[k \rho^{2}+T^{2}\right]^{1 / 2}} .
\end{aligned}
$$

The boundary conditions corresponding to (2.17) and (2.18) are

$$
\begin{aligned}
\left.e_{\theta}\left(k\left[k \rho^{2}+T^{2}\right]^{1 / 2}, \frac{d}{d \rho} \rho T\right)\right|_{\rho=1} & =\frac{\mu}{a}=\frac{\lambda}{a} k, \\
\frac{k\left[k \rho^{2}+T^{2}\right]^{1 / 2}}{1+e_{\theta}\left(k\left[k \rho^{2}+T^{2}\right]^{1 / 2}, k d(\rho T) / d \rho\right)_{\rho=1}} & =\sigma=s k
\end{aligned}
$$

and $T$ should be finite at $\rho=0$. The quantities $\lambda$ and $s$ are defined by (3.7) and (3.8).

The functions $e_{r}\left(\Sigma_{r}, \Sigma_{\theta}\right)$ and $e_{\theta}\left(\Sigma_{r}, \Sigma_{\theta}\right)$ (cf. (3.3)) may be rewritten in terms of $T$

$$
\begin{aligned}
e_{r}\left(k\left(k \rho^{2}+T^{2}\right)^{1 / 2}, k \frac{d}{d \rho}(\rho T)\right) & \\
= & \frac{k}{E}\left[\frac{d}{d \rho}(\rho T)-\nu\left(k \rho^{2}+T^{2}\right)^{1 / 2}\right] \\
& +\frac{k^{2}}{E^{2}}\left\{\nu\left(k \rho^{2}+T^{2}\right)-(1-\nu)\left(k \rho^{2}+T^{2}\right)^{1 / 2} \frac{d}{d \rho}(\rho T)-\nu^{2}\left(\frac{d}{d \rho}(\rho T)\right)^{2}\right\} \\
& +\vartheta\left(k^{3}\right), \\
e_{\theta}\left(k \left(k \rho^{2}\right.\right. & \left.\left.+T^{2}\right)^{1 / 2}, k \frac{d}{d \rho}(\rho T)\right) \\
= & \frac{k}{E}\left\{\frac{d}{d \rho}(\rho T)-\nu\left(k \rho^{2}+T^{2}\right)^{1 / 2}\right\} \\
& +\frac{k^{2}}{E^{2}}\left\{\nu\left(\frac{d}{d \rho}(\rho T)\right)^{2}-(1-\nu)\left(k \rho^{2}+T^{2}\right)^{1 / 2} \frac{d}{d \rho}(\rho T)-\nu^{2}\left(k \rho^{2}+T^{2}\right)\right\} \\
& +\vartheta\left(k^{3}\right) .
\end{aligned}
$$


It is a consequence of (3.9) that

$$
\begin{aligned}
& \frac{e_{r}\left(k\left(k \rho^{2}+T^{2}\right)^{1 / 2}, k d(\rho T) / d \rho\right)}{k}=\frac{1}{E}\left(T-\nu \frac{d}{d \rho}(\rho T)\right)+\vartheta(k), \\
& \frac{e_{\theta}\left(k\left(k \rho^{2}+T^{2}\right)^{1 / 2}, k d(\rho T) / d \rho\right)}{k}=\frac{1}{E}\left(\frac{d}{d \rho}(\rho T)-\nu T\right)+\vartheta(k) .
\end{aligned}
$$

In addition

$$
\frac{1-T /\left(k \rho^{2}+T^{2}\right)^{1 / 2}}{k}=\frac{\rho^{2}}{2 T^{2}}+\vartheta(k) .
$$

It is a consequence of (3.10) and (3.11) that (3.6) can be written

$$
\frac{d}{d \rho} \frac{\rho}{E}\left(\frac{d}{d \rho}(\rho T)-\nu T\right)+\frac{\rho^{2}}{2 T^{2}}-\frac{1}{E}\left(T-\nu \frac{d}{d \rho}(\rho T)\right)+\vartheta(k)=0 .
$$

Equation (3.12) can be rearranged to show that the equation which is accurate to $\theta(k)$ is

$$
\frac{d^{2} T}{d \rho^{2}}+\frac{3}{\rho} \frac{d T}{d \rho}+\frac{E}{2 T^{2}}=0
$$

Equation (3.13) is the Foppl equation. Indeed there is no difficulty in putting (3.13) into the exact form used in either [3] or [4] and [5]. The boundary conditions on (3.13) accurate to $\vartheta(k)$ are determined from either (3.7)

$$
\left.\frac{1}{E}\left(\frac{d}{d \rho}(\rho T)-\nu T\right)\right|_{\rho=1}=\lambda / a
$$

or (3.8)

$$
T(1)=s .
$$

In both cases we require that $T(0)$ should be finite. The conditions (3.14) and (3.15) agree exactly with the boundary conditions usually prescribed in the Foppl theory.

In principle there is no difficulty in finding theories accurate to higher order. Indeed expansions of $e_{r}$ and $e_{\theta}$ accurate to $\theta\left(k^{2}\right)$ can be obtained from (3.9). The remaining terms in the equation can also be determined to $\theta\left(k^{2}\right)$. As a consequence (3.6) and both the boundary conditions (3.7) and (3.8) can be explicitly determined to $\vartheta\left(k^{2}\right)$.

4. Appendix. The exact equation. In this section we will derive the equations (1.1) and (1.3). A point in the circular membrane with coordinates $(x, y, 0)$ has a new position $(\xi, \eta, \zeta)$ after deformation. In polar coordinates we may write

$$
(r \cos \theta, r \sin \theta, 0) \rightarrow((r+u) \cos \theta,(r+u) \sin \theta, w)
$$

where $u$ is the radial displacement and $w$ is the vertical displacement. The assumption of radial symmetry implies that $u=u(r)$ and $w=w(r)$, i.e. both $u$ and $w$ are independent of $\theta$.

In order to determine the strains we note that as a result of the deformation an element of length $d S$ before deformation will be transformed into an element of length $d S^{*}$ after 
deformation where

$$
\begin{gathered}
(d S)^{2}=(d r)^{2}+r^{2}(d \theta)^{2} \\
\left(d S^{*}\right)^{2}=\left[\left(1+u^{\prime}\right)^{2}+\left(w^{\prime}\right)^{2}\right](d r)^{2}+\left(1+\frac{u}{r}\right)^{2} r^{2}(d \theta)^{2} .
\end{gathered}
$$

It is convenient to choose the relative extensions in the radial and circumferential directions as the definition of the radial and circumferential strains (the symmetry assumption implies that the shear strains and shear stresses vanish). Thus we find

$$
\begin{aligned}
& \varepsilon_{r}=\frac{\left[\left(1+u^{\prime}\right)^{2}+\left(w^{\prime}\right)^{2}\right]^{1 / 2} d r-d r}{d r}, \\
& \varepsilon_{\theta}=\frac{\left(1+\frac{u}{r}\right) r d \theta-r d \theta}{r d \theta}
\end{aligned}
$$

The equations (1.1) are an immediate consequence.

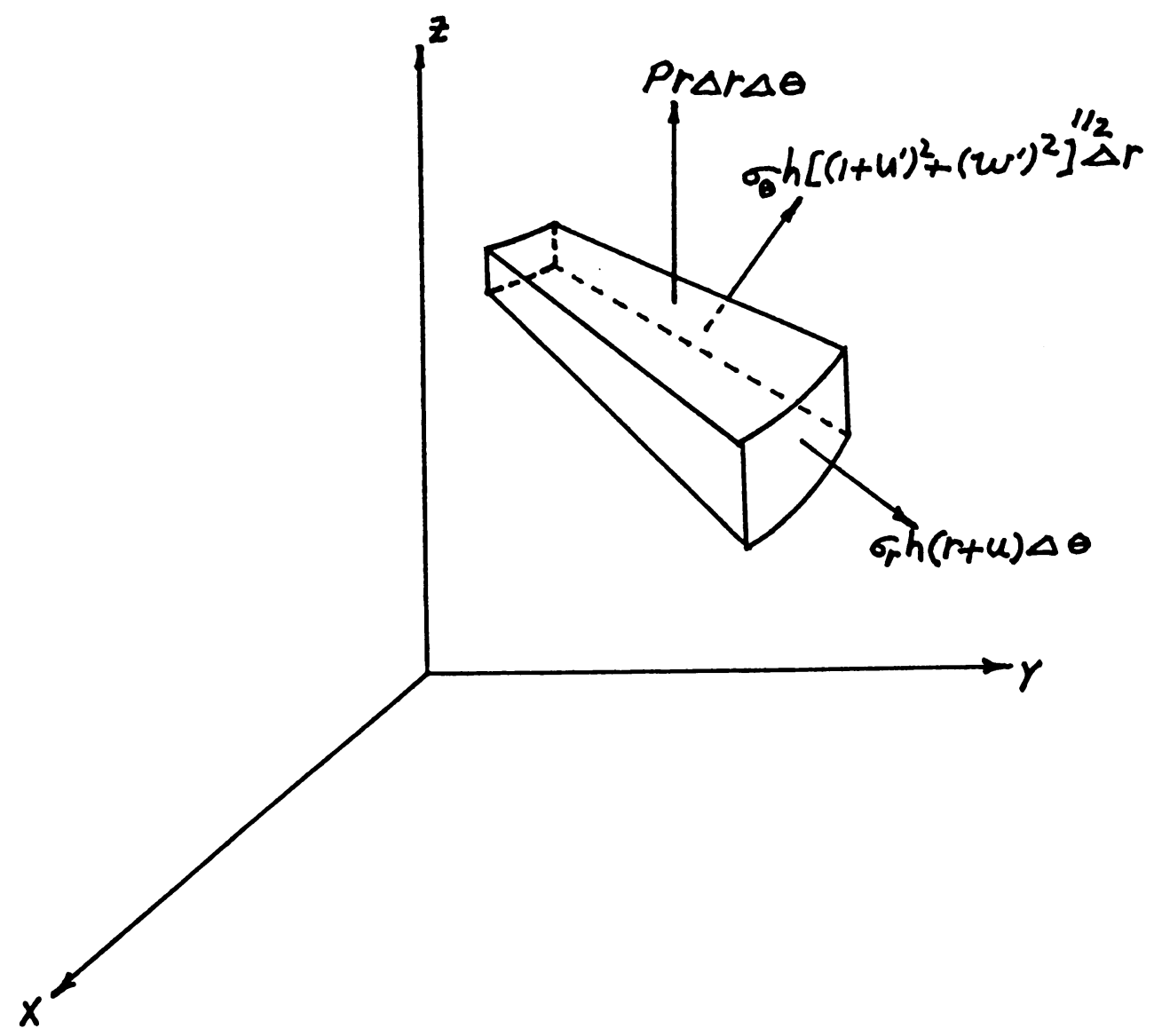

FIG. 1 
In Fig. 1 we have drawn an element of the deformed membrane and indicated the magnitude of the faces on the positive faces (the quantities are defined in Sec. 1). The unit normal to a face $r=$ constant is

$$
\frac{\left(1+u^{\prime}\right) \bar{r}+w^{\prime} \bar{k}}{\left[\left(1+u^{\prime}\right)^{2}+\left(w^{\prime}\right)^{2}\right]^{1 / 2}}
$$

and the unit normal to the face $\theta=$ constant is $\bar{\theta}$ where

$$
\begin{aligned}
& \bar{r}=\cos \theta \bar{i}+\sin \theta \bar{j}, \\
& \bar{\theta}=-\sin \theta \bar{i}+\cos \theta \bar{j}
\end{aligned}
$$

with $\bar{i}, \bar{j}, \bar{k}$ the unit vectors in the $x, y$, and $z$ directions. The equilibrium conditions become

$$
\left.\frac{\sigma_{r} h(r+u)\left[\left(1+u^{\prime}\right) \bar{r}+w^{\prime} \bar{k}\right]}{\left[\left(1+u^{\prime}\right)^{2}+\left(w^{\prime}\right)^{2}\right]^{1 / 2}} \Delta \theta\right|_{r} ^{r+\Delta r}+\left.\sigma_{\theta} h\left[\left(1+u^{\prime}\right)^{2}+\left(w^{\prime}\right)^{2}\right]^{1 / 2} \bar{\theta} \Delta r\right|_{\theta} ^{\theta+\Delta \theta}
$$

Dividing (4.8) by $h \Delta r \Delta \theta$ and taking the limit as $\Delta r \rightarrow 0$ and $\Delta \theta \rightarrow 0$ we find

$$
\frac{d}{d r}\left\{\frac{\sigma_{r}(r+u)\left[\left(1+u^{\prime}\right) \bar{r}+w^{\prime} \bar{k}\right]}{\left[\left(1+u^{\prime}\right)^{2}+\left(w^{\prime}\right)^{2}\right]^{1 / 2}}\right\}-\sigma_{\theta}\left[\left(1+u^{\prime}\right)^{2}+\left(w^{\prime}\right)^{2}\right]^{1 / 2} \bar{r}+\frac{\operatorname{Pr} \bar{k}}{h}=0 .
$$

The equations (1.3) are an immediate consequence of (4.9).

\section{REFERENCES}

[1] A. Foppl, Vorlesungen über technische Mechanik, Bd. 5, P. Teubner, Leipzig, 1907

[2] H. Hencky, Über der Spannungszustand in kreisrunden Platten, Z. Math. Phys. 63, 311-317 (1915)

[3] R. W. Dickey, The plane circular elastic surface under normal pressure, Arch. Rational Mech. Anal. 26, 219-236 (1967)

[4] A. J. Callegari and E. L. Reiss, Nonlinear boundary value problems for the circular membrane, Arch. Rational Mech. Anal. 31, 390-400 (1970)

[5] A. J. Callegari, H. B. Keller, and E. L. Reiss, Membrane buckling: A study of solution multiplicity, Comm. Pure Appl. Math. 24, 499-521 (1971) 